\title{
Co-administration of THC and MDMA ('Ecstasy') Synergistically Disrupts Memory in Rats
}

\author{
June M Young', lain S McGregor ${ }^{2}$ and Paul E Mallet*, \\ 'School of Psychology, University of New England, Armidale, NSW, Australia; ${ }^{2}$ School of Psychology, University of Sydney, NSW, Australia
}

\begin{abstract}
3,4-Methylenedioxymethamphetamine (MDMA, 'Ecstasy') and cannabis are two of the most commonly used illicit drugs in the western world, and are often used in combination. Very little research has examined their effect on cognitive function or behavior when combined. The present study used a double $Y$-maze task to examine the acute effect of MDMA and $\Delta^{9}$-tetrahydrocannabinol (THC, the principal psychoactive ingredient of cannabis) on mnemonic function in rats, at a range of doses representative of common human use. Experiment I (low doses) examined the effect of $0.25 \mathrm{mg} / \mathrm{kg}$ THC and $1.25 \mathrm{mg} / \mathrm{kg}$ MDMA alone and together. At these doses MDMA or $\mathrm{THC}$ given alone had no effect on working memory, but the co-administered drugs significantly disrupted working memory. Experiment 2 (medium doses) examined the effect of $0.5 \mathrm{mg} / \mathrm{kg}$ THC and $2.5 \mathrm{mg} / \mathrm{kg}$ MDMA given alone or together. At these doses THC, but not MDMA, impaired working memory. Although MDMA alone had no effect, it exacerbated the impairment due to THC when the drugs were co-administered. Experiment 3 (high doses) examined the effects of I mg/kg THC and $5 \mathrm{mg} / \mathrm{kg}$ MDMA alone and together. Both drugs significantly impaired memory when given alone, although the impairment due to MDMA was less than that caused by THC. When co-administered at these doses, the drugs caused a major disruption of behavior and this precluded ascribing a mnemonic cause to poor performance on the double Y-maze task. Taken together, these experiments demonstrate a synergistic disruption of working memory by acute co-administration of THC and MDMA.

Neuropsychopharmacology (2005) 30, 1475-1482, advance online publication, 16 February 2005; doi: 10.1038/sj.npp. 1300692
\end{abstract}

Keywords: N-methyl-3,4-methylenedioxyamphetamine; tetrahydrocannabinol; drug interactions; memory; maze learning; rats

\section{INTRODUCTION}

3,4-Methylenedioxymethamphetamine (MDMA, 'Ecstasy') is a substituted amphetamine whose popularity has risen to make it one of the four most commonly used illicit drugs in the world (Christophersen, 2000; Smart and Ogborne, 2000). It is typically taken by young people at dance parties for its effects of euphoria, increased energy, sensuality, and a feeling of closeness to others (Downing, 1986; Verheyden et al, 2003). Elation, however, is often accompanied by anxiety and confusion (Tancer and Johanson, 2003), and other undesirable side effects include minor appetite loss, dryness of mouth, and bruxism, plus a characteristic period of depression, irritability, and impaired concentration lasting several days, which begins a day or two after taking the drug (Parrott and Lasky, 1998; Verheyden et al, 2003).

The acute physiological and emotional effects of MDMA in humans and animals have been well documented (eg Cole and Sumnall, 2003; Parrott et al, 2000; Tancer and Johanson,

\footnotetext{
*Correspondence: Dr PE Mallet, School of Psychology, University of New England, Armidale, NSW 235I, Australia, Tel: + 6126773 3725, Fax: + 61 26773 3820, E-mail: paul.mallet@une.edu.au Received 20 May 2004 and accepted 28 December 2004 Online publication: 12 January 2005 at http://www.acnp.org/citations/ Npp0 I I 205040235/default.pdf
}

2003; Vollenweider et al, 1998), but acute effects on cognition appear complex and less clear. In laboratory animals, short-term memory, color discrimination, and position discrimination were not affected by acute MDMA (Frederick and Paule, 1997; Ricaurte et al, 1993), while time estimation, motivation, and learning were impaired. Braida et al (2002) found no effect of MDMA on acute short-term memory in rats, but subacute memory was affected (see also Barrionuevo et al, 2000). In human studies, MDMA users have demonstrated verbal memory impairments both while on-drug (Parrott and Lasky, 1998; Parrott et al, 1998) and after a history of ecstasy use, while other cognitive functions appeared normal (Bolla et al, 1998; Reneman et al, 2000). Recently, however, attention has been drawn to the important distinctions between effects in human adults and adolescents (Jacobsen et al, 2004), and to the realization that many studies of human ecstasy users have not controlled sufficiently for poly-drug use (Roiser and Sahakian, 2003).

Indeed, poly-drug use appears to be the norm for ecstasy users, with up to $93 \%$ of ecstasy users falling into this category (Schifano et al, 2003; Smart and Ogborne, 2000; Topp et al, 1999; Turner et al, 2003; Winstock et al, 2001). Cannabis is the illicit drug most commonly co-used with ecstasy, presumably because of its overall high popularity 
and also its reputed effect of reducing the unpleasant aftereffects (Topp et al, 1999). An Australian survey (Topp et al, 1999 ) reported that $45 \%$ of MDMA users took cannabis concomitantly with MDMA, and $64 \%$ used cannabis when 'coming down' from MDMA. Winstock et al (2001) reported $82 \%$ of MDMA users in the UK using cannabis both concomitantly with ecstasy and to 'come down'. Cannabis itself is the most commonly used illicit drug in the western world. Its principal psychoactive ingredient is $\Delta^{9}$-tetrahydrocannabinol (THC, Gaoni and Mechoulam, 1964). In humans and in animals, cannabis or cannabinoid receptor agonists generally alter mood and impair short-term memory (for a review, see Ameri, 1999).

While the individual acute effects of MDMA and THC are well documented, their combined effect has received little attention. The two drugs exert their effects through differing neurotransmitter systems. THC primarily acts through cannabinoid receptors located in several brain areas, with particularly high densities found in outflow nuclei of the basal ganglia, the hippocampus, and the cerebellum (Herkenham et al, 1990). Cannabinoid receptor activation produces several effects, including stimulation of mesoprefrontal dopaminergic transmission (Diana et al, 1998) and enhanced dopamine release in the nucleus accumbens (Chen et al, 1991). MDMA exerts its unique combination of behavioral and mood effects by widespread activation of the brain's serotonin, dopamine, and norepinephrine systems (Climko et al, 1986). It is therefore conceivable that the combination of MDMA and THC may produce additive, subtractive, or synergistic effects. While a small number of studies have addressed their long-term interaction in humans (Croft et al, 2001; Daumann et al, 2003; Gouzoulis-Mayfrank et al, 2000), acute specific interactions between the two drugs are yet to be documented. The present study therefore aimed to investigate the combined effect of acute administration of MDMA and THC on a major component of cognitive function, namely memory, in rats. Specifically, it sought to document the effects of the two drugs coadministered, across a range of low to moderate doses that are representative of human use (Boot et al, 2000).

Mnemonic function was tested in young adult rats using a double Y-maze task which provides a valid assessment of memory and includes control procedures that guard against misinterpretation of possible non-mnemonic effects including altered motivation, sensory-perceptual abilities, and motor function (Mallet and Beninger, 1993; Mason et al, 1999; Smith et al, 1994).

\section{MATERIALS AND METHODS}

All experimental procedures were carried out in accordance with NIH and NHMRC guidelines (NIH Publications No. 80-23, revised 1996; NHMRC Code of Practice 1997, respectively), and were approved by the University of New England Animal Ethics Committee.

\section{Subjects}

A total of 40 male albino Wistar rats (University of New England Animal House) weighing $190-275 \mathrm{~g}$ at the beginning of the study were group-housed in a temperature- controlled room $\left(21 \pm 1^{\circ} \mathrm{C}\right)$ maintained on a $12 \mathrm{~h}$ reversed light cycle (lights on $1900 \mathrm{~h}$ ). Animals had free access to water throughout the study. After a 3-day handling period, food was removed from the group cages and animals were maintained thenceforth on a food-restricted program to maintain the animals at $80-85 \%$ of their free-feeding weights, adjusted for growth.

\section{Drugs}

MDMA (Australian Govt Analytical Laboratories, Pymble, NSW) was dissolved in $0.9 \%$ saline. THC $(10 \mathrm{mg} / \mathrm{ml}$ dissolved in ethanol, Sigma-Aldrich, Sydney, Australia) was mixed with Tween 80 (polyoxyethylene sorbitan monooleate, ICN Biomedicals, $1 \%$ of final vehicle). The ethanol was evaporated under a stream of nitrogen gas and the resulting suspension was mixed with $0.9 \%$ saline to the required concentration. Both drugs were administered i.p. in a volume of $1 \mathrm{ml} / \mathrm{kg}$ bodyweight. Drug doses $(0.25,0.5$, $1.0 \mathrm{mg} / \mathrm{kg}$ THC; $1.25,2.5,5.0 \mathrm{mg} / \mathrm{kg}$ MDMA) were chosen to represent low, moderate, and high equivalent doses in humans (Boot et al, 2000).

\section{Apparatus}

Memory was assessed using a double Y-maze (Mallet and Beninger, 1993). The maze (Figure 1) was constructed of white melamine and was placed on a $70-\mathrm{cm}$-high table. All passages were $16.5 \mathrm{~cm}$ wide with walls $25.5 \mathrm{~cm}$ high. Each of the four end arms was $36 \mathrm{~cm}$ long and extended at $120^{\circ}$ from a 54-cm-long central stem. Doors slid vertically into batten slots to form boxes at the end of each arm or barriers at either end of the central stem. An opaque food cup was placed at the end of each arm and in the central stem. Small pieces of Froot Loops breakfast cereal (Kelloggs) were

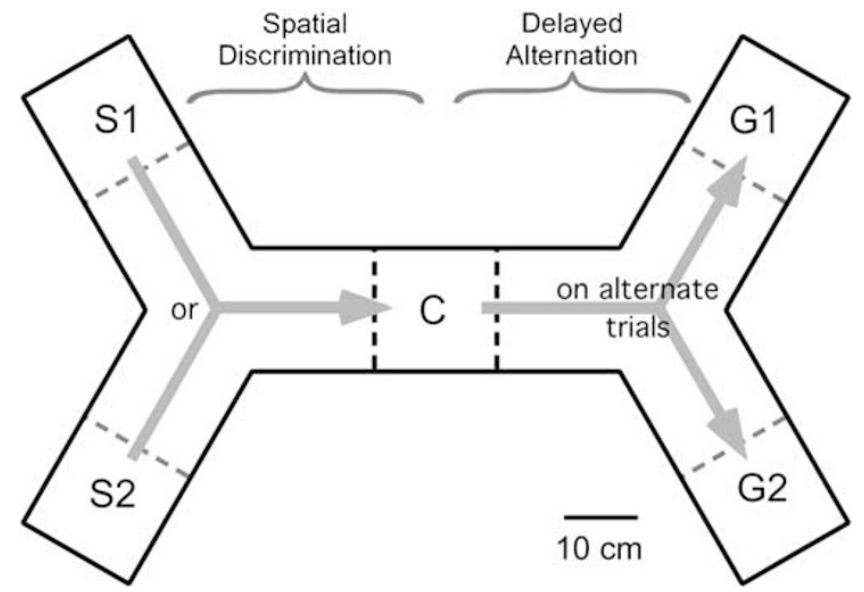

Figure I Schematic representation of a top view of the double Y-maze. Broken lines represent the locations of removable barriers that were used to restrict access to different areas of the maze. Plastic food cups were placed in all goal boxes. Each trial began by placing a rat in one of the two start boxes, $\mathrm{SI}$ or S2; half the trials began in box SI and the other half began in box S2. Boxes SI and SI never contained food; hence, the correct spatial discrimination choice was to choose the central box C. Once reinforced in box $C$, animals were given access to the second (right) $Y$ maze. Food was available in either goal box GI or G2; the correct delayed alternation choice was to choose the box that had not been visited on the preceding trial. Arrows show correct paths leading to reinforcement. 
placed in the cups to reward correct responses; the cereal could not be seen inside the cups from a distance, and cereal dust was sprinkled throughout the maze to mask any odor cues. The apparatus was housed in a dedicated temperature-controlled room with moderate white lighting (300 lux). From within the maze, every arm was visually identical; distinctive external visual cues (posters, lights, researcher) were visible from within the maze. A pink noise generator continually masked ambient laboratory noise. The maze was cleaned with a commercial glass-cleaning solution after each session.

\section{Design}

Three experiments using identical testing protocols but different drug doses were conducted. Each experiment used a separate group of drug-naïve rats and a within-subjects design. In each experiment, rats received four treatments: (1) vehicle, (2) THC alone, (3) MDMA alone, and (4) THC + MDMA combined. In Experiment 1 (low dose, $N=16$ ), the doses used were $0.25 \mathrm{mg} / \mathrm{kg}$ THC and $1.25 \mathrm{mg} / \mathrm{kg}$ MDMA. In Experiment 2 (medium dose, $N=16$ ), doses for THC and MDMA were 0.5 and $2.5 \mathrm{mg} / \mathrm{kg}$, respectively. In Experiment 3 (high dose, $N=8$ ), doses for THC and MDMA were 1.0 and $5.0 \mathrm{mg} / \mathrm{kg}$, respectively. The order of treatments was counterbalanced across animals in each experiment according to a Latin square design.

\section{Behavioral Testing}

The double Y-maze was developed as a valid measure of mnemonic function, avoiding some of the possible confounds of the traditional radial arm maze (Mallet and Beninger, 1993). In essence, the double Y-maze presents the rat with two consecutive tasks on each trial: a spatial discrimination task in the first ' $\mathrm{Y}$ ', followed by a delayed alternation task in the second ' $\mathrm{Y}$ ' (see Figure 1). In trained rats, the spatial discrimination task is a test of reference (trial independent) memory, while the delayed alternation task is a test of reference memory plus working (trial dependent) memory. The tasks make identical demands on the rat in all ways (eg motivation, locomotion, sensory perception), except for the addition of the working memory component of the second task. Thus, if a trained rat performs as expected in the first task of the maze (involving reference memory only), then poorly in the second task (involving reference and working memory), the difference can confidently be attributed to a failure in working memory (Mallet and Beninger, 1993).

Habituation. Rats were extensively handled twice daily for 3 days. The rats were then individually habituated to the maze on each day for four consecutive days. During each habituation session, the rats were given 10 min free access to all parts of the maze to collect cereal rewards from the food cups, and were gradually introduced to the movement of doors around them until they showed no overt signs of anxiety within the maze.

Training. Training was then conducted over the next 2 and one half weeks. The training procedure was slightly modified from that described by Mason et al (1999). After habituation, each rat received one training session of 25 trials per day. For each rat, the start box and initial goal box were randomly allocated for each session to prevent the rats from using algorithmic maze-solving strategies. Rats ran both halves of the maze on each trial, concurrently learning a different task in each ' $Y$ ': a spatial discrimination task in the first ' $\mathrm{Y}$ ', and a delayed alternation task in the second ' $\mathrm{Y}$ '. Rats learnt both tasks in approximately the same number of trials (data not shown), suggesting that the tasks were roughly equivalent in difficulty.

To train the animals, each rat was placed randomly into the start box 'S1' or 'S2', with the door at the distal end of the centre box (C) closed (see Figure 1). When the start box door was opened, the rat's task was to always proceed directly into box $\mathrm{C}$, without entering the other start box. With its distal door closed, box $\mathrm{C}$ appeared identical to each start box, so the rat had to learn to use external visual cues to solve this first ' $\mathrm{Y}$ ' maze. This portion of the maze task therefore presented a spatial discrimination task. If the rat entered the wrong arm, no reward was available but no intervention took place; rather, the rat was allowed to proceed until it located the reward in box $\mathrm{C}$. When the rat had entered box $\mathrm{C}$ and collected the reward, its retreat back into the first ' $\mathrm{Y}$ ' was blocked by placing a door behind the rat (at the proximal end of $\mathrm{C}$ ), and the distal door of box $\mathrm{C}$ was then removed.

The rat was now faced with a second ' $\mathrm{Y}$ ', whose arms again were visually identical. This time the task was to choose the alternate arm on each trial, irrespective of the original start arm (S1 or S2): in other words, if the reward was placed in the first goal box (G1) on the first trial, it was now placed in G2 on the second trial, G1 on the third trial, and so on. For the first trial of each session, the initial goal box (G1 or G2) was randomly allocated. Initially, the correct alternating route to the rewarded arm was forced by blocking the other arm. When the rat had learnt to turn quickly and confidently enter the correct, unblocked arm on each trial (after approximately 1 week of training), the alternating block was discontinued after Trial 1 and the rat was left free to choose the goal arm. On a correct choice, the rat received its cereal reward, then was returned immediately to a start box to commence another trial. If the rat entered an incorrect arm, it was not rewarded and was returned immediately to the same start box to run that trial again until it found the reward. The training criterion was set at three or fewer errors, totaled across both tasks, in each session of 24 scored trials, for two consecutive sessions. The rats learnt rapidly to alternate accurately to receive their rewards, reaching the training criterion in approximately another 1 week. The first trial of each session was additional to the scored trials: it was not scored and was always blocked (in random order for each session) to prevent the rat from determining its own pattern of retrieval and to force genuine mnemonic processing in the alternation task.

When rats had reached the training criterion, they were familiarized with the injection procedure and the introduction of delays in the start boxes. Delays were imposed by confining the rat to the start box for 0,15 , or $60 \mathrm{~s}$ at the beginning of each trial. Approximately $4 \mathrm{~s}$ was required between each trial to return the rat to the start box, re-bait cups, and position the doors. The real time between 
consecutive choices should therefore be understood as 0,15 , or $60 \mathrm{~s}$ plus approximately $4 \mathrm{~s}$. Delays were allocated in random order, with the provisos that an equal number of each delay (ie eight) occurred during each session, and no one length of delay was imposed more than three times consecutively. Similarly, start boxes (S1 or S2) were allocated randomly for each trial, with the provisos that an equal number of each start box was included (ie 12) in each session, and no start box was used more than three times consecutively. The initial goal box was also randomly allocated for each session, with the proviso that an equal number of each initial goal box was allocated each day. These procedures together ensured that (a) rats could not develop an algorithmic pattern of responding, or responses based on odor trails, and (b) there was no connection between the start box, length of delay, or correct choice of goal arm.

Testing. Before test sessions, rats received two injections: THC or its vehicle, followed 10 min later by MDMA or its vehicle. The control condition involved two vehicle injections only. Trials began $20 \mathrm{~min}$ after the second injection. Test sessions were identical to the final, delay-inclusive training sessions. A trial was scored as correct if the rat directly entered the required arm. An incorrect trial was scored as such, then repeated (without further scoring) until the correct arm was entered. Although this meant that poor performance entailed more (unscored) trials than good performance, the overall percentage of extra trials was in fact minimal and would not be expected to impact per se on results.

After each drug session, rats were retrained drug-free to a criterion of three or less errors within a single session. Retraining began the day following drug treatment. The criterion was regained by $84 \%$ of the rats after one retraining session, and the remaining rats gained criterion after two retraining sessions. Notably, longer retraining was not necessarily required after administration of the highdose combination which was behaviorally debilitating, but tended to pertain more to individual learning patterns of the animals. Drug treatments were reinstated the day after reaching the retraining criterion, and were thus never administered less than $48 \mathrm{~h}$ apart to any rat. There was no systematic difference between any drug groups in retraining requirements or subsequent length of time between drug treatments.

\section{Data Analysis}

All raw results were converted to percentage correct choices. Data were analyzed separately for each task component by two-way (treatment by delay) repeatedmeasures ANOVA using SPSS version 11. Where significant main effects were observed, pairwise comparisons were conducted using Bonferroni-adjusted t-tests. Mauchley's test of sphericity was violated in the low-dose condition for within-subjects effect of delay, but application of the Huynh-Feldt adjustment did not alter the results. The results can therefore be viewed as being robust against violations of the sphericity assumption. To further substantiate this point, data were re-analyzed using a twofactor nonparametric randomization test of scores using
NPFact version 1.0 (May et al, 1993). In all cases the results of the randomization tests agreed with the ANOVA results, so for ease of interpretation only the ANOVA tests are shown, which reflect the Huynh-Feldt correction.

To determine whether THC and MDMA interacted synergistically, data were also analyzed using three-way (delay by MDMA by THC) repeated-measures ANOVAs. In these analyses there were three levels of delay: 0,15 , and $60 \mathrm{~s}$. The factors MDMA and THC were each allocated two levels: presence or absence. A separate three-way repeatedmeasures ANOVA was conducted for the delayed alternation and spatial discrimination data at the low and medium doses. The high-dose data were not available for the combined treatment condition (see below); these data were therefore not subjected to three-way analysis. Although the three-way ANOVAs each yielded seven F tests (three main effects and four interactions), only the THC by MDMA, and the three-way interaction were examined, as the remaining $F$ tests did not provide any important information not already provided by the two-way ANOVAs.

\section{RESULTS}

\section{Delayed Alternation Task}

Response accuracy in the delayed alternation component declined as the delay interval was increased across all treatments and all experiments (Figure 2). The two-way ANOVAs revealed a significant main effect of delay in the low-, medium-, and high-dose experiments, $\mathrm{F}(2,30)=158.88$, $p<0.001, \mathrm{~F}(2,30)=45.23, p<0.001$, and $\mathrm{F}(2,14)=44.33$, $p<0.001$, respectively.

In the low-dose experiment (Figure 2a), MDMA and THC alone had no appreciable effect on response accuracy. However, the combined administration of these drugs resulted in a decline in response accuracy at the 15- and 60 -s delays. The delay by treatment ANOVA revealed a significant main effect of treatment, $F(3,45)=22.99$, and a significant treatment by delay interaction, $\mathrm{F}(6,90)=3.28$, $p<0.01$. Pairwise comparisons revealed that the combined drug treatment was significantly different from all other treatments, $p<0.001$. There was no significant difference between the vehicle, THC alone, and MDMA alone treatments.

The low-dose three-way ANOVA yielded a significant three-way (delay by THC by MDMA) interaction, $\mathrm{F}(2,30)=4.44, p<0.05$. The THC by MDMA interaction was also significant, $\mathrm{F}(1,15)=33.64, p<0.001$. These results indicate that the two drugs interacted synergistically to impair choice accuracy in the delayed alternation, but this effect was dependent on the delay interval. Examination of Figure 2(a) shows that choice accuracy was increasingly impaired by the combined drug treatment relative to all other treatments as the delay interval was increased.

In the medium-dose experiment (Figure 2b), MDMA alone had no effect relative to vehicle at the 0 - and 15-s delays, but a slight increase in response accuracy was observed at the 60-s delay. Administration of THC alone resulted in a substantial decrease in response accuracy at all delays. The combination of THC and MDMA led to a large reduction in response accuracy at all delays tested. The delay by treatment ANOVA revealed a significant main 

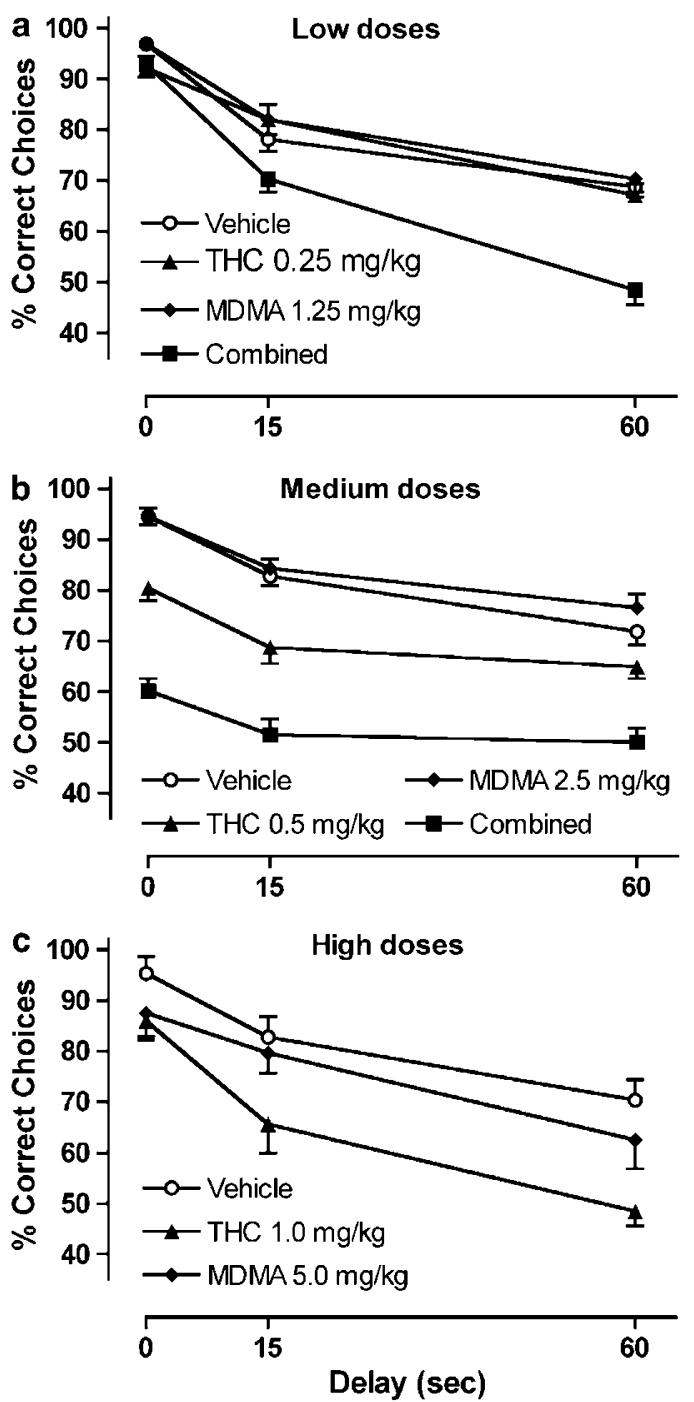

Figure 2 Mean ( + or - SEM) percentage of correct responses in the delayed alternation task across three delay intervals following administration of vehicle, MDMA, THC, or MDMA + THC combined. Respective doses for THC and MDMA were 0.25 and $1.25 \mathrm{mg} / \mathrm{kg}$ in the low-dose experiment (a), 0.5 and $2.5 \mathrm{mg} / \mathrm{kg}$ in the medium-dose experiment (b), and 1.0 and $5.0 \mathrm{mg} / \mathrm{kg}$ in the high-dose experiment (c).

effect of treatment, $F(3,45)=74.64, p<0.001$, but the interaction was not significant. Pairwise comparisons revealed that THC impaired performance relative to both vehicle and MDMA alone, while the combined drug treatment impaired memory performance more than any other treatment, $p<0.001$.

The three-way (delay by THC by MDMA) interaction for the medium dose data was not significant, $\mathrm{F}(2,30)=0.39$, $p>0.05$. However, the THC by MDMA interaction was significant, $\mathrm{F}(1,15)=42.04, p<0.0001$. These results indicate that the two drugs synergistically reduced choice accuracy in the delayed alternation in a delay-independent manner.

In the high-dose experiment (Figure 2c), MDMA alone produced a small decrease and THC alone produced a substantial decrease in response accuracy at all delays. When THC and MDMA were combined, a profound behavioral impairment that prevented the rats from successfully completing either task within the maze was evident, so no data are available for the combined treatment. Locomotor ability per se did not appear to be affected, but rather than moving purposefully through the maze the rats turned in circles, shuttled backwards and forwards, lay down, or rolled onto their backs for some time. Several rats paid particular attention to their genital area to the exclusion of other activities. Cereal rewards were sometimes eaten when found, or sometimes ignored. Performance in both tasks of the maze was so disrupted that errors could not be confidently ascribed to a deficit of memory, and the results of the high dose combined treatment group were therefore excluded from the analysis.

The two-way ANOVA conducted on the remaining highdose data revealed a significant main effect of treatment, $\mathrm{F}(2,15)=30.00, p<0.001$, but the treatment by delay interaction was not significant. Pairwise comparisons revealed that THC and MDMA alone both impaired performance compared to the vehicle treatment, $p<0.05$. Furthermore, THC impaired memory performance more than MDMA, $p<0.01$.

\section{Spatial Discrimination Task}

Performance in the spatial discrimination component was highly accurate across all treatments and delays in the lowdose (Figure 3a) and medium-dose (Figure 3b) experiments. In the high dose experiment (Figure 3c), rats receiving combined administration of THC and MDMA were unable to complete the maze task, so no data were available for analysis. A separate two-way (treatment by delay) analysis of variance (ANOVA) with repeated measures on both factors was used to compare groups in each experiment. None of the analyses resulted in a significant main effect of treatment, main effect of delay, or treatment by delay interaction $(P>0.05)$. The three-way ANOVAs also did not yield any significant three-way, or MDMA by THC interactions.

\section{DISCUSSION}

The results of the present study add to a growing body of evidence that THC acutely impairs memory (Essman, 1984; Heyser et al, 1993; Lichtman and Martin, 1996; Mallet and Beninger, 1998; Varvel et al, 2001). In addition, results revealed that MDMA alone did not significantly affect memory at the low or medium doses tested, but MDMA at these doses interacted with THC to produce an impairment of memory that was greater than that observed with THC alone. The results of the three-way ANOVAs revealed that MDMA and THC acted synergistically to impair memory in a delay-dependent manner for the low doses, and in a delayindependent manner for the medium doses. To our knowledge, this is the first report that MDMA potentiates THC-induced memory impairment in a supra-additive manner.

Experiment 1 (low doses) yielded particularly interesting results. Here, subthreshold doses of THC or MDMA alone did not impair memory. Combined, however, THC and MDMA significantly impaired working memory, which was evidenced by impaired choice accuracy in the delayed 


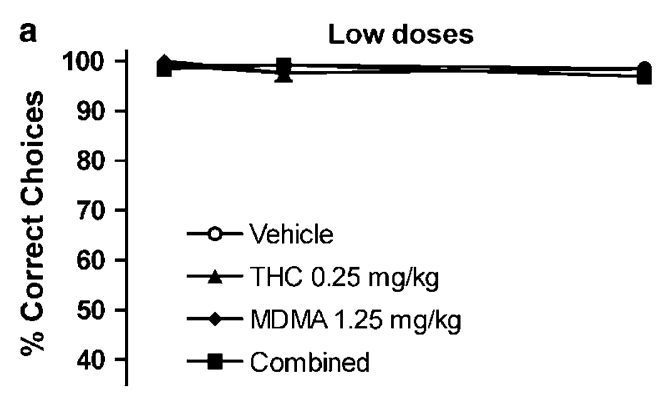

either maze task such that the performance deficit could not be confidently interpreted as a mnemonic effect.

The spatial discrimination component of the double $\mathrm{Y}$ maze requires the use of reference memory only, whereas the delayed alternation component also requires the use of working memory (Mallet and Beninger, 1993). Our findings with THC are therefore in good agreement with previous studies (eg Mallet and Beninger, 1996, 1998; Nakamura et al, 1991), showing that THC impairs working, but not reference, memory. At low to moderate doses, the memory-impairing effects of THC and THC + MDMA were specific to the delayed alternation component of the task; that is, the spatial discrimination component was unaffected. As both components of the double Y-maze make equal demands on non-mnemonic variables (eg motor function, motivation, perceptual abilities), it can be confidently concluded that the drug-induced decrease in choice accuracy was due to impaired working memory.

The administration of MDMA alone did not affect choice accuracy at low or medium doses, and impairment at high dose was modest. Braida et al (2002) found that acute administration of up to $3 \mathrm{mg} / \mathrm{kg}$ MDMA initially had no effect on the number of errors in a standard eight-arm radial maze task, but when a 2 -h delay was imposed, MDMA dose-dependently impaired performance. Similarly, administration of MDMA $(10 \mathrm{mg} / \mathrm{kg})$ prior to acquisition of a passive avoidance task impaired memory assessed $24 \mathrm{~h}$ later (Barrionuevo et al, 2000). The studies by Braida et al, and Barrionuevo et al, employed much longer delays than those reported here. Whether MDMA would impair choice accuracy with longer delay intervals in the double Y-maze remains an open question.

The neurochemical basis for the observed synergistic effect of the combined drugs is an interesting matter for speculation. Evidence regarding direct interaction of cannabinoid and serotonergic systems in impairment of memory is very limited (Egashira et al, 2002; Molina-Holgado et al, 1995). However, as well as exerting its effects via activation of $\mathrm{CB}_{1}$ receptors, THC is known to increase dopamine production in several areas including prefrontal mesocortical areas strongly connected with working memory function (Bergson et al, 2003; Jentsch et al, 1997; Pistis et al, 2002). MDMA has direct action on the serotonin, dopamine, and norepinephrine neurochemical systems (Climko et al, 1986), suggesting that an interaction of the two drugs may occur within the dopamine system. Both THC and MDMA are also known to strongly affect cholinergic release in areas critically involved in acute mnemonic function (Acquas et al, 2001a,b; Fischer et al, 2000; Nava et al, 2001), and their combination may have a complex effect on its production. Both dopaminergic and cholinergic results may apply not only in prefrontal cortex, but in hippocampal regions - particularly the dentate gyrus - which may compromise navigational function in tandem with short-term memory function (Egashira et al, 2002; Shankaran and Gudelsky, 1998; White et al, 1996; Hermann et al, 2002).

In conclusion, this study found that acutely co-administered THC and MDMA in rats, within a dose range relevant to human consumption, produced an impairment of working memory greater than that of either MDMA or THC alone. It was particularly notable that subthreshold 
doses of the two individual drugs produced an observable impairment in working memory when combined. While this acute effect was robust, there was no indication from this study whether the impairment would be long lasting, or extend to other cognitive processes. The neurochemical mechanisms underlying this result are presently a matter of speculation but represent an interesting direction for further research.

\section{ACKNOWLEDGEMENTS}

This study was supported by an Australian Research Council Grant to ISM and PEM and an NH\&MRC grant to ISM. JMY's PhD studies were supported by an Australian Postgraduate Award.

\section{REFERENCES}

Acquas E, Marrocu P, Pisanu A, Cadoni C, Zernig G, Saria A et al (2001a). Intravenous administration of ecstasy (3,4-methylenedioxymethamphetamine) enhances cortical and striatal acetylcholine release in vivo. Eur J Pharmacol 418: 207-211.

Acquas E, Pisanu A, Marrocu P, Goldberg SR, di Chiara G (2001b). $\Delta^{9}$-tetrahydrocannabinol enhances cortical and hippocampal acetylcholine release in vivo: a microdialysis study. Eur J Pharmacol 419: 155-161.

Ameri A (1999). The effects of cannabinoids on the brain. Progr Neurobiol 58: 315-348.

Barrionuevo M, Aguirre N, Del Rio J, Lasheras B (2000). Serotonergic deficits and impaired passive-avoidance learning in rats by MDEA: a comparison with MDMA. Pharmacol Biochem Behav 65: 233-240.

Bergson C, Levenson R, Goldman-Rakic PS, Lidow MS (2003). Dopamine receptor-interacting proteins: the $\mathrm{Ca}^{2+}$ connection in dopamine signaling. Trends Pharmacol Sci 24: 486-492.

Bolla KI, McCann UD, Ricaurte GA (1998). Memory impairment in abstinent MDMA ('Ecstasy') users. Neurology 51: 1532-1537.

Boot BP, McGregor IS, Hall W (2000). MDMA (Ecstasy) neurotoxicity: assessing and communicating the risks. Lancet 355: 1818-1821.

Braida D, Pozzi M, Cavallini R, Sala M (2002). 3,4-Methylenedioxymethamphetamine (Ecstasy) impairs eight-arm radial maze performance and arm entry pattern in rats. Behav Neurosci 116: 298-305.

Chen J, Paredes W, Lowinson JH, Gardner EL (1991). Strainspecific facilitation of dopamine efflux by $\Delta^{9}$-tetrahydrocannabinol in the nucleus accumbens of rat: an in vivo microdialysis study. Neurosci Lett 129: 175-179.

Christophersen AS (2000). Amphetamine designer drugs - an overview and epidemiology. Toxicol Lett 112: 127-131.

Climko RP, Roehrich H, Sweeney DR, Al-Razi J (1986). Ecstasy: a review of MDMA and MDA. Int J Psychiatry Med 16: 359-372.

Cole JC, Sumnall HR (2003). The pre-clinical behavioural pharmacology of 3,4-methylenedioxymethamphetamine (MDMA). Neurosci Biobehav Rev 27: 199-217.

Croft RJ, Mackay AJ, Mills ATD, Gruzelier JGH (2001). The relative contributions of ecstasy and cannabis to cognitive impairment. Psychopharmacology 153: 373-379.

Daumann J, Schnitker R, Weidemann J, Schnell K, Thron A, Gouzoulis-Mayfrank E (2003). Neural correlates of working memory in pure and polyvalent ecstasy (MDMA) users. NeuroReport 14: 1983-1987.

Diana M, Melis M, Gessa GL (1998). Increase in meso-prefrontal dopaminergic activity after stimulation of $\mathrm{CB} 1$ receptors by cannabinoids. Eur J Neurosci 10: 2825-2830.
Downing J (1986). The psychological and physiological effects of MDMA on normal volunteers. J Psychoactive Drugs 18: 335-340.

Egashira N, Mishima K, Katsurabayashi S, Yoshitake T, Matsumoto Y, Ishida J et al (2002). Involvement of 5hydroxytryptamine neuronal system in $\Delta^{9}$-tetrahydrocannabinol-induced impairment of spatial memory. Eur J Pharmacol 445: 221-229.

Essman EJ (1984). Marijuana intoxication in rats: interruption of recent memory and effect on brain concentration of delta-9tetrahydrocannabinol. Psychol Bull 55: 563-567.

Fischer HS, Zernig G, Schatz DS, Humpel C, Saria A (2000). MMA ('ecstasy') enhances basal acetylcholine release in brain slices of the rat striatum. Eur J Neurosci 12: 1385-1390.

Frederick DL, Paule MG (1997). Effects of MDMA on complex brain function in laboratory animals. Neurosci Biobehav Rev 21: 67-78.

Gaoni Y, Mechoulam R (1964). Isolation, structure, and partial synthesis of an active constituent of hashish. J Am Chem Soc 86: 1646-1647.

Gouzoulis-Mayfrank E, Daumann J, Tuchtenhagen F, Pelz S, Becker S, Kunert H-J et al (2000). Impaired cognitive performance in drug free users of recreational ecstasy (MDMA). J Neurol Neurosurg Psychiatry 68: 719-725.

Herkenham M, Lynn AB, Little M, Johnson R, Melvin L, de Costa BR et al (1990). Cannabinoid receptor localisation in brain. Proc Natl Acad Sci USA 87: 1932-1936.

Hermann H, Marsicano G, Lutz B (2002). Coexpression of the cannabinoid receptor type 1 with dopamine and serotonin receptors in distinct neuronal subpopulations of the adult mouse forebrain. Neuroscience 109: 451-460.

Heyser CJ, Hampson RE, Deadwyler SA (1993). Effects of delta-9tetrahydrocannabinol on delayed match to sample performance in rats: alterations in short-term memory associated with changes in task specific firing of hippocampal cells. J Pharmacol Exp Therap 264: 294-307.

Jacobsen LK, Mencl WE, Pugh KR, Skudlarski P, Krystal JH (2004). Preliminary evidence of hippocampal dysfunction in adolescent MDMA ('ecstasy') users: possible relationship to neurotoxic effects. Psychopharmacology 173: 383-390.

Jentsch JD, Andrusiak E, Tran A, Bowers Jr MB, Roth RH (1997). $\Delta^{9}$-Tetrahydrocannabinol increases prefronta lcortical catecholaminergic utilization and impairs spatial working memory in the rat: blockade of dopaminergic effects with HA 966. Neuropsychopharmacology 16: 426-432.

Lichtman AH, Martin BR (1996). $\Delta^{9}$-Tetrahydrocannabinol impairs spatial memory through a cannabinoid receptor mechanism. Psychopharmacology 126: 125-131.

Mallet PE, Beninger RJ (1993). The double Y-maze as a tool for assessing memory in rats. Neurosci Protocols 93: 01-11.

Mallet PE, Beninger RJ (1996). The endogenous cannabinoid receptor agonist anandamide impairs memory in rats. Behav Pharmacol 7: 276-284.

Mallet PE, Beninger RJ (1998). The cannabinoid $\mathrm{CB}_{1}$ receptor antagonist SR141716A attenuates the memory impairment produced by $\Delta^{9}$-tetrahydrocannabinol or anandamide. Psychopharmacology 140: 11-19.

Mason KI, Mallet PE, Jhamandas K, Boegman RJ, Beninger RJ (1999). Nucleus basalis injections of $N$-methyl-D-aspartate enhance memory of rats in the double Y-maze. Brain Res Bull 48: $65-71$.

May R, Hunter M, Gabriel R (1993). NPFact 1.0. http://home. utah-inter.net/rmay/npstat.html.

Molina-Holgado F, Gonzalez MI, Leret ML (1995). Effect of $\Delta^{9}$ -tetrahydrocannabinol on short-term memory in the rat. Physiol Behav 57: 177-179.

Nakamura EM, da Silva EA, Concilio GV, Wilkinson DA, Masur J (1991). Reversible effects of acute and long-term 
administration of $\Delta$-9-tetrahydrocannabinol (THC) on memory in the rat. Drug Alcohol Depend 28: 167-175.

Nava F, Carta G, Colombo G, Gessa GL (2001). Effects of chronic $\Delta^{9}$-tetrahydrocannabinol treatment on hippocampal extracellular acetylcholine concentration and alternation performance in the T-maze. Neuropharmacology 41: 392-399.

Parrott AC, Lasky J (1998). Ecstasy (MDMA) effects upon mood and cognition: before, during and after a Saturday night dance. Psychopharmacology 139: 261-268.

Parrott AC, Lees A, Garnham NJ, Jones M, Wesnes K (1998). Cognitive performance in recreational users of MDMA or 'ecstasy': evidence for memory deficits. J Psychopharmacol 12: 79-83.

Parrott AC, Sisk E, Turner JJD (2000). Psychobiological problems in heavy 'ecstasy' (MDMA) polydrug users. Drug Alcohol Depend 60: 105-110.

Pistis M, Ferraro L, Pira L, Flore G, Tanganelli S, Gessa GL et al (2002). $\Delta^{9}$-Tetrahydrocannabinol decreases extracellular GABA and increases extracellular glutamate and dopamine levels in the rat prefrontal cortex: an in vivo microdialysis study. Brain Res 948: 155-158.

Reneman L, Booij J, Schmand B, van den Brink W, Gunning B (2000). Memory disturbances in 'Ecstasy' users are correlated with an altered brain serotonin neurotransmission. Psychopharmacology 148: 322-324.

Ricaurte GA, Markowska AL, Wenk GL, Hatzidimitriou G, Wlos J, Olton DS (1993). 3,4-Methylenedioxymethamphetamine, serotonin and memory. J Pharmacol Exp Therap 266: 1097-1105.

Roiser JP, Sahakian BJ (2003). Relationship between ecstasy use and depression: a study controlling for poly-drug use. Psychopharmacology 173: 411-417.

Schifano F, Oyefeso A, Corkery J, Cobain K, Jambert-Gray R, Martinotti $G$ et al (2003). Death rates from ecstasy (MDMA, MDA) and polydrug use in England and Wales 1996-2002. Hum Psychopharmacol Clin Exp 18: 519-524.
Shankaran M, Gudelsky GA (1998). Effect of 3,4-methylenedioxymethamphetamine (MDMA) on hippocampal dopamine and serotonin. Pharmacol Biochem Behav 61: 361-366.

Smart RG, Ogborne AC (2000). Drug use and drinking among students in 36 countries. Addict Behav 25: 455-460.

Smith CG, Beninger RJ, Mallet PE, Jhamandas K, Boegman RJ (1994). Basal forebrain injections of the benzodiazepine partial inverse agonist FG 7142 enhance memory of rats in the double Y-maze. Brain Res 666: 61-67.

Tancer M, Johanson C-E (2003). Reinforcing, subjective, and physiological effects of MDMA in humans: a comparison with $d$ amphetamine and mCPP. Drug Alcohol Depend 72: 33-44.

Topp L, Hando J, Dillon P, Roche A, Solowij N (1999). Ecstasy use in Australia: patterns of use and associated harm. Drug Alcohol Depend 55: 105-115.

Turner C, Russell A, Brown W (2003). Prevalence of illicit drug use in young Australian women, patterns of use and associated risk factors. Addiction 98: 1419-1426.

Varvel SA, Hamm RJ, Martin BR, Lichtman AH (2001). Differential effects of $\Delta^{9}$ THC on spatial reference and working memory in mice. Psychopharmacology 157: 142-150.

Verheyden SL, Henry JA, Curran HV (2003). Acute, sub-acute and long-term subjective consequences of 'ecstasy' (MDMA) consumption in 430 regular users. Hum Psychopharmacol Clin Exp 18: $507-517$.

Vollenweider FX, Gamma A, Liechti M, Huber T (1998). Psychological and cardiovascular effects and short-term sequelae of MDMA ('Ecstasy') in MDMA-naive healthy volunteers. Neuropsychopharmacology 19: 241-251.

White SR, Obradovic KM, Imel KM, Wheaton MJ (1996). The effects of methylenedioxymethamphetamine (MDMA, 'Ecstasy') on monoaminergic neurotransmission in the central nervous system. Progr Neurobiol 49: 455-479.

Winstock AR, Griffiths P, Stewart D (2001). Drugs and the dance music scene: a survey of current drug use patterns among a sample of dance music enthusiasts in the UK. Drug Alcohol Depend 64: 9-17. 